\title{
Mapping Laboratory Reports to Illinois' Extensively Drug-Resistant Organism Registry
}

\author{
Emily Augustini ${ }^{\star 1}$, William Trick ${ }^{2}$ and Stacey Hoferka ${ }^{1}$ \\ 'Office of Health Protection, Division of Infectious Diseases, Illinois Department of Public Health, Chicago, IL, USA; ${ }^{2}$ Cook County \\ Health \& Hospitals System, CDC Prevention Epicenters Program, Chicago, IL, USA
}

\section{Objective}

To streamline carbapenem-resistant enterobacteriaceae (CRE) surveillance by integrating electronic laboratory reporting (ELR) data and electronic case reports (eCR) automatically into Illinois' extensively drug-resistant organism (XDRO) registry.

\section{Introduction}

CRE are drug-resistant bacteria that have a mortality rate of up to $50 \%$ in those infected ${ }^{1}$. Several clusters of CRE have been detected in Illinois, often in long-term acute care hospitals ${ }^{2}$. In response Illinois created the XDRO registry, a mandatory reporting system designed to aid inter-facility communication concerning CRE.

Despite being a high priority for control in the US, the case definition for CRE has been the subject of debate ${ }^{3}$. There are over 70 Enterobacteriaceae which can have different mechanisms of carbapenem resistance ${ }^{3}$. Criteria for carbapenem resistance include susceptibility results, and phenotypic or genotypic detection. The case definition for the XDRO registry is intentionally more exclusive (specific) than that used by CSTE (Table 1). CSTE utilizes a definition designed to maximize sensitivity. Illinois' XDRO registry's definition is more specific, meant to reduce unnecessary adoption of contact precautions and the negative consequences some patients may experience.

Currently, case reporting to the XDRO registry is a manual data entry process, which has important advantages. However, transitioning to automatic ELR integration will streamline the reporting process and minimize data entry effort. Unfortunately, the clinical information needed to investigate XDROs is often not captured by ELR. The eCR is a new message type being piloted in Illinois that contains many clinical data elements. We examined the feasibility of combining ELR and $\mathrm{eCR}$ into reports for the XDRO registry. In the construction of these reports we examined the impact of using CRE definitions from CSTE and the XDRO registry.

\section{Methods}

We obtained sample HL7 CRE messages from Illinois' ELR database. Using these messages and the HL7 Implementation Guide for Electronic Laboratory Reporting, we mapped ELR fields to those in the XDRO registry. Specific codes corresponding to the registry fields were found though a systematic keyword search of LOINC, SNOMED, and sample messages. When there was no match for an XDRO field in ELR, we referred to the HL7 CDA Implementation Guide for the Electronic Initial Case Report and sample eCR messages. A collection of fields and codes was created to correspond to both the CSTE and Illinois CRE case definition.

\section{Results}

The XDRO registry has 37 unique fields. Twenty-six can be populated from ELR, four can be found in the eCR, and seven are generated within the system. In sample ELR and eCR messages all of the necessary fields were populated with appropriate text and codes. The mapping process was straightforward for demographic and facility information, but more complicated for culture and organism information. Some XDRO tests do not have corresponding LOINC or SNOMED codes, so we will develop a logic statement to fill these based on free-text. Addition of the eCR adds important information to the registry report, notably encounter type and encounter/admission date. We were able to create separate mapping schemas for the CSTE and XDRO registry definitions for CRE. Using each of these definitions, we will quantify how many ELR messages would be committed to the XDRO registry.

\section{Conclusions}

By combining the data captured in ELR and eCR, it is possible to populate the fields of the Illinois XDRO registry. When this merge is completed it should result in more complete and better quality data on CRE in Illinois. As intended, the definition of CRE used by the registry is less inclusive than that used by CSTE. Future work will show the number of CRE lab results captured by each definition.

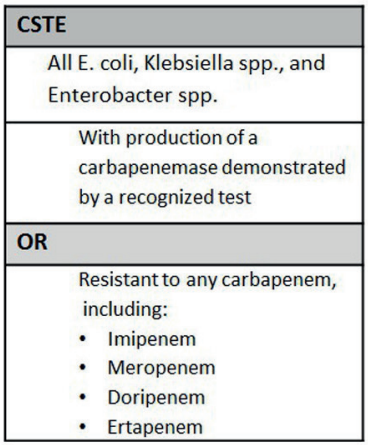

\begin{tabular}{|c|}
\hline XDRO Registry \\
\hline All Enterobacteriaceae \\
\hline $\begin{array}{l}\text { With a positive molecular or } \\
\text { phenotypic test for carbapenemase }\end{array}$ \\
\hline OR \\
\hline E. coli or Klebsiella species \\
\hline $\begin{array}{l}\text { Non-susceptible to one of the } \\
\text { following: } \\
\text { - Imipenem } \\
\text { - Meropenem } \\
\text { - Doripenem }\end{array}$ \\
\hline AND \\
\hline Resistant to all third generation \\
cephalosporins tested
\end{tabular}

Table 1: CRE Definition

Keywords

CRE; Data Mapping; ELR; eCR

\section{Acknowledgments}

Project SHINE

\section{References}

1. Centers for Disease Control and Prevention (CDC). (2015). CRE Toolkit: Facility Guidance for Control of Carbapenem-resistant Enterobacteriaceae. Atlanta, Georgia: CDC.

2. Trick, W. E., Lin, M. Y., Cheng-Leidig, R., Driscoll, M., Tang, A. S., Gao, W., Weinstein, R. A. (2015). Electronic Public Health Registry of Extensively Drug-Resistant Organisms, Illinois, USA. Emerging Infectious Diseases. 21(10):1725-1732.

3. Council of State and Territorial Epidemiologists (CSTE). (2015). Standardized definition for Carbapenem-resistant Enterobacteriaceae (CRE) and recommendation for sub-classification and stratified reporting. 15-ID-05.

\section{*Emily Augustini}

E-mail: emily.augustini@illinois.gov 